\title{
Etapas del proceso de planificación estratégica en las empresas mixtas petroleras
}

\author{
Stages of the strategic planning process in mixed oil companies \\ Etapas do processo de planejamento estratégico em empresas petrolíferas mistas
}

Recibido: enero 2019

Arbitrado: febrero 2019

Publicado: mayo 2019

\author{
《rossana Garcia \\ rossanagarcia30@gmail.com \\ ORCID: 0000-0001-9331-8023
}

Universidad del Zulia, Venezuela

\section{RESUMEN}

La presente investigación tuvo como objetivo identificar las etapas del proceso de planificación estratégica en las empresas mixtas petroleras del municipio Maracaibo Venezuela. Metodológicamente se tipifico como una investigación descriptiva, con un diseño no experimental, transeccional y de campo. La población quedo constituida por los gerentes de los departamentos de perforación de las empresas mixtas del municipio Maracaibo. La técnica de recolección de datos fue la encuesta y el instrumento un cuestionario con cinco alternativas de respuesta, estructurado por 31 ítems, se utilizó el juicio de expertos para la validez del mismo y el coeficiente Alfa Cronbach para la confiabilidad, cuyo resultado fue 0,96. La información fue procesada a través de la estadística descriptiva específicamente mediante la media aritmética. Se concluye que moderadamente las empresas mixtas analizadas conocen e implementan la formulación de estrategias, ejecución de estrategias y evaluación de estrategias como etapas del proceso de la planificación estratégica.

Palabras clave: Ejecución de estrategias; etapas del proceso; evaluación de estrategias; formulación de estrategias, planificación estratégica
The objective of this investigation was to identify the stages of the strategic planning process in the joint oil companies of the Maracaibo municipality. Methodologically it was typified as a descriptive investigation, with a non-experimental, cross-sectional and field design. The population was made up of the managers of the drilling departments of the mixed companies of the Maracaibo municipality. The data collection technique was the survey and the instrument a questionnaire with five response alternatives, structured by 31 items, expert judgment was used for its validity and the Alpha Cronbach coefficient for reliability, whose result was 0 , 96. The information was processed through descriptive statistics specifically using the arithmetic mean. It is concluded that the analyzed joint ventures moderately know and implement the formulation of strategies, execution of strategies and evaluation of strategies as stages of the strategic planning process.

Key words: Strategy execution; stages of the process; evaluation of strategies; strategy formulation, strategic planning
RESUMO

O objetivo desta pesquisa foi identificar as etapas do processo de planejamento estratégico nas empresas petrolíferas mistas do município de Maracaibo, Venezuela Metodologicamente, foi tipificada como uma pesquisa descritiva, com desenho não experimental, transversal e de campo. A população era formada pelos gestores dos departamentos de perfuração das empresas mistas do município de Maracaibo. A técnica de coleta de dados foi o survey e o instrumento um questionário com cinco alternativas de respostas, estruturado em 31 itens, utilizou-se o julgamento de especialistas para sua validade e o coeficiente Alpha de Cronbach para confiabilidade, cujo resultado foi 0,96 . As informações foram processadas por meio de estatística descritiva especificamente por meio da média aritmética. Conclui-se que as joint ventures analisadas têm moderadamente conhecimento e implementam a formulação de estratégias, execução de estratégias e avaliação de estratégias como etapas do processo de planejamento estratégico.

Palavras chave: Estratégia de execução; etapas do processo; avaliação de estratégias; formulação de estratégia, planejamento estratégico 


\section{INTRODUCCIÓN}

$\mathrm{L}$ a mayoría de los cambios gestados en los países latinoamericanos y en las empresas tanto privadas como públicas se debe a la incorporación de nuevas tecnologías que aceleran la competitividad de los mercados haciendo variar las estrategias y objetivos de cada empresa con tal rapidez que no permite el logro de algunos objetivos cuando ya estos son cambiados. De manera tal, en la lucha de estas empresas por mejorar los procesos existentes y la capacidad del mercado mundial surge la necesidad de adaptar soluciones estratégicas para desarrollar el mayor potencial posible a sus usuarios.

En este sentido, para cualquier empresa, la planificación estratégica puede ser una herramienta que ayude alcanzar cualquier objetivo que se desee lograr. Este tipo de planificación es entendida según Goodstein y otros (2006), como el proceso por el cual los miembros guías de una organización prevén su futuro y desarrollan los procedimientos y operaciones necesarias para alcanzar sus objetivos.

Desde esta perspectiva, la planificación estratégica incrementa la capacidad de la organización para incrementar el plan estratégico de manera completa y oportuna. Así mismo, ayuda a que la organización desarrolle, organice y utilice una mejor comprensión del entorno en el cual opera, o la industria o campo donde funciona, de sus clientes (actuales y potenciales) y de sus propias capacidades y limitaciones.

En este punto las empresas en Venezuela no deberían quedar relegadas del resto del mundo. Las empresas y organizaciones públicas y privadas deben ocuparse en el desarrollo de nuevas formas para mejorar la optimización de los procesos; es así, como dentro del contexto organizacional la planificación estratégica no solo tiene que ver con decisiones futuras, por el contrario, está relacionada con la toma de decisiones actuales que afectaran a la organización en el futuro.

Cabe destacar que las empresas mixtas petroleras del municipio Maracaibo no escapan de esta realidad, es por ello que se puede analizar la eficiencia de estas organizaciones según la planificación estratégica que estas tengan. Tales instituciones, orientándose según los valores presentes en las filosofías de gestión que las sustentan, deben justificar su espacio en la sociedad a la que atienden, dando cuenta de la inversión/gasto que representan en relación directa con los resultados/beneficios que producen. De allí que a través de una adecuada planificación estratégica los líderes de estas organizaciones logren aumentar de forma significativa la productividad de sus procesos, logrando con esto, cumplir en gran parte con los objetivos trazados.

Es de hacer notar que en municipio Maracaibo, las empresas mixtas petroleras, en el departamento de perforación, tratan de aplicar planificación estratégica donde se obtengan buenos resultados. Sin embargo la observación directa por parte del investigador, además de algunas entrevistas directas e informales al personal involucrado en el área objeto de estudio, 
evidencian que algunas de las empresas mixtas no poseen un criterio alineado para desarrollar las estrategias planificadas, cada una de ellas posee su estilo o forma en particular, por lo que surgen dificultades de diversa índole, entre los que se encuentran: duplicidad de tareas, improvisación, pérdida de tiempo, desorganización, y caos, entre otras que genera atraso en la atención de los requerimientos realizados por los usuarios.

Por ello, se requiere desarrollar un análisis de planificación estratégica basada en las ya aplicadas exitosamente a nivel mundial en este tipo de empresas a fin de identificar las etapas del proceso de planificación estratégica, que permita orientar con facilidad a los usuarios para el desarrollo de dicha estrategia y buscar lograr los objetivos y metas propuestas, todo apuntando a mantener la rentabilidad y eficiencia del negocio.

\section{Planificación estratégica}

Las empresas no trabajan sobre la base de la improvisación, para Chiavenato (2005), la planificación figura como la primera función administrativa, siendo ésta la base de las demás funciones. La misma, determina anticipadamente cuáles son los objetivos a alcanzar y qué hacer para alcanzarlos, se trata de un modelo teórico para las acciones futuras.

Adicionalmente, Münch y García (2008), expresan que la planificación precede a las demás etapas del proceso administrativo, ya que planear implica hacer la elección de las decisiones más adecuadas acerca de lo que se habrá de realizar en el futuro. En cuanto a la estrategia, Hax y Majluf (2004), indican que se trata de un proceso formal de la planificación, que evoluciona a partir de pautas amplias hasta planes concretos de acción.

Por otra parte, Serna (2008), menciona que la planificación estratégica es el proceso mediante el cual quienes toman decisiones en una organización obtienen, procesan y analizan información pertinente, interna y externa; con el fin de evaluar la situación presente de la empresa, así como el nivel de competitividad con el propósito de anticipar y decidir sobre el direccionamiento de la institución hacia el futuro.

Esta consideración sobre la planificación estratégica se enfoca como un proceso que tiene como primer paso el planteamiento de estrategias gerenciales y operativas para que las organizaciones puedan alcanzar sus objetivos y lograr las metas establecidas a largo plazo. De esta manera, la planificación estratégica es un proceso participativo que se caracteriza por identificar estrategias de actuación, a partir de análisis internos y externos, con las cuales una organización se adapta a los cambios y demandas que le impone el entorno para lograr el máximo de eficiencia y calidad de sus productos o servicios. 


\section{Etapas del proceso de planificación estratégica}

En criterio de Borrell (2003), las etapas del proceso de planificación estratégica deben basarse en los siguientes puntos: información, que es el proceso de detección de una amenaza o una oportunidad (FODA); estudio, es la búsqueda de cursos de acción alternativos que sirvan para resolver el problema creado o para aprovechar la ocasión que se presenta; valoración, que consiste en hacer una estimación de las consecuencias de cada una de las alternativas; decisión; que consiste en la elección de una de las alternativas (o no tomar ninguna de ellas, no hacer nada). Las ideas para nuevas estrategias pueden surgir en cualquier lugar de la organización; todos los miembros pueden tener una idea brillante $o$ al menos el germen de idea.

En su obra "conceptos de administración estratégicas", David (2008), describe "las etapas del proceso de planificación estratégica indicando que existe una etapa de formulación, ejecución y evaluación, las cuales se conjugan para determinar la forma de planificar el futuro de las organizaciones"(p.5). Para efectos de este trabajo, se considera este planteamiento por cuanto especifica cada uno de los elementos integrantes de las etapas mencionadas, lo cual permite orientar las acciones estratégicas en el área. A los efectos de conocer el punto de vista de los autores, y en especial de David (2008) se especifica de la siguiente manera:

\section{Formulación de estrategias}

De acuerdo a David (2008), la formulación puede definirse como la primera etapa de la planificación estratégica conducente a la fijación de la misión de la firma, llevando a cabo una investigación con el objetivo de establecer las debilidades, fortalezas oportunidades y amenazas externas, realizando análisis que comparen factores internos y externos, fijando objetivos y estrategias para la empresa.

Los gerentes de alto nivel poseen la mejor perspectiva para comprender en su totalidad los distintos aspectos de las decisiones de formulación, además de poseer la autoridad para comprometer los recursos necesarios para la implantación. Esta etapa en afirmaciones hechas por David (2008), incluye la creación de una misión y visión, identificación de las oportunidades y amenazas externas de una organización, la determinación de las fortalezas y debilidades internas, el establecimiento de objetivos a largo plazo y la generación de estrategias alternativas. Esto significa, que para bien o para mal, las decisiones estratégicas producen consecuencias importantes en diversas funciones y efectos duraderos en una organización.

Este proceso de administración estratégica es vital para las empresas mixtas, desde su inicio, todas las organizaciones cuentan con una estrategia, aunque la estrategia solo sea el resultado de las operaciones diarias, el proceso, incluso realizado de manera informal, puede marcar una gran diferencia en el crecimiento y la prosperidad en las organizaciones. 
En opinión de Certo (2008), esta etapa consiste en el proceso de diseño de una estrategia que pueda dar lugar a una ventaja competitiva sostenible; una vez que la gerencia ha analizado el ambiente y fijado una dirección para la organización, están en condiciones de trazar estrategias competitivas en un esfuerzo bien documentado para mejorar las posibilidades de éxito de la organización.

En este sentido, Serna (2008), plantea que las opciones estratégicas se convierten en planes de acción concretos con definición de responsables para lograrlos. Para ello es indispensable programar en el tiempo proyectos estratégicos, definir objetivos y las estrategias de cada área funcional de la organización, así como diseñar planes de acción concretos para ser consolidados en función de los propósitos establecidos de la institución.

En cuanto a las referencias empleadas para describir esta etapa, se aprecia en cuanto al resultado de esta etapa puede dar origen a numerosas ventajas competitivas, sin embargo David (2008), hace un completo esbozo del proceso que aquí se lleva a cabo, señalando que allí la misión y visión son desarrolladas; Certo (2008) y Serna (2008), por su parte explica que esta etapa permite hacer proyecciones en el tiempo.

A efectos de la investigación, se debe abordar esta concepción estratégica, ya que se ha demostrado que el conocer y compartir la misión y visión de una organización, mejora el desempeño de la institución, de igual manera la planificación y determinación de objetivos influyen positivamente en el esfuerzo que las personas imprimen a su trabajo. Para el caso de este estudio, se consideran los postulados de David (2008), quien plantea el análisis interno y externo, descrito a continuación:

Análisis interno: Según expresa David (2008), este comienza por una auditoria que se base en la participación de todos los gerentes y empleados de la empresa para determinar las fortalezas y las debilidades de la misma, a través de la recolección y asimilación de información sobre las operaciones de dirección, logística, mercadotecnia, finanzas y contabilidad, producción y operaciones, investigación y desarrollo y sistemas de información de la gerencia de la empresa, de tal manera que las fortalezas y las debilidades más importantes de la empresa se determinen de manera colectiva.

Asimismo Serna (2008), considera que el análisis interno de la empresa se base en un perfil de capacidad interna de la organización $(\mathrm{PCl})$, el cual es un medio para evaluar las fortalezas y debilidades de la compañía en relación con las oportunidades y amenazas que le presenta el medio externo, involucrando todos los factores que afectan la operación corporativa de la empresa.

Por lo tanto, para lograr el análisis interno de la organización es importante efectuar auditorías periódicas que consistan en evaluar todas las capacidades internas de la empresa, con la finalidad de conocer la situación de la organización respecto a cada uno de los factores descritos anteriormente. 
Análisis externo: Opinan Thompson y Strickland (2007) que el análisis externo comprende la revisión de las variables que rodean el ambiente organizacional, incluyendo oportunidades y amenazas. Las oportunidades son aquellos factores que resultan positivos, favorables, explotables, que se deben descubrir en el entorno en el que actúa la empresa, y que permiten obtener ventajas competitivas. Dentro de las oportunidades deben considerarse el comportamiento de la empresa en el mercado, la competitividad, así como las tendencias políticas, sociales, económicas y tecnológicas.

En este mismo orden de ideas, para David (2008), el análisis externo consiste en definir a través de una auditoria externa, las oportunidades que podrían beneficiar a una empresa y las amenazas que deben evitarse. A tales efectos el objetivo de la auditoría externa es identificar las principales variables que ofrezcan respuestas prácticas. Las empresas deben responder a los factores de manera tanto ofensiva como defensiva, por medio de la formulación de estrategias que aprovechen las oportunidades externas o que reduzcan el impacto de las amenazas potenciales.

De igual manera Serna (2008), coincide con las definiciones descritas con anterioridad sobre el análisis externo empresarial, y lo plantea como un factor determinante para enfrentar oportunamente en forma dinámica y acelerada los desafíos de los cambios, por ejemplo, los cambios de gustos de los clientes, de las condiciones políticas, de la estructura del mercado, así como los tecnológicos.

Visión: Para David (2008), se debe responder a la pregunta básica; qué se quiere llegar a ser. Una visión definida proporciona el fundamento para crear una declaración de la misión integral. Muchas empresas poseen tanto la declaración de la visión como la de la misión, pero la declaración de la visión debe establecerse en primer lugar. La declaración de la visión debe ser corta, formada de preferencia por una oración y desarrollada por tantos gerentes como sea posible.

Según Serna (2008), la visión es un conjunto de ideas generales, algunas de ellas abstractas, que proveen el marco de referencia de lo que una empresa es y quiere ser en el futuro. La visión no se expresa en términos numéricos, la define la alta dirección de la compañía, debe ser amplia e inspiradora, conocida por todos e integrar al equipo gerencial a su alrededor, requiere líderes para su definición y para su cabal realización.

De la misma forma, la visión señala el rumbo, da dirección, sirve de guía en la formulación de las estrategias, a la vez que le proporciona un propósito a la organización. La visión debe reflejarse en la misión, los objetivos y las estrategias de la institución y se hace tangible cuando se materializa en proyectos y metas específicas, cuyos resultados deben ser medibles mediante un sistema de índices de gestión bien definidos. Por ello, el ejercicio de definir la visión es una tarea gerencial de mucha importancia, de allí que sea parte fundamental del proceso estratégico de una empresa. 
En este sentido, las distintas definiciones establecidas anteriormente acerca de la visión, guardan correspondencia entre sí, y sus fundamentos deben ser difundida en la organización y reconocida, por ello requiere un sistema de difusión que la haga conocer y comprender por todos los miembros de la organización.

Misión: Thompson y Strickland (2007) afirman que la misión es el motivo, propósito, fin o razón de ser de la existencia de una empresa u organización por que define:(1) lo que pretende cumplir en su entorno o sistema social en el que actúa, (2) lo que pretende hacer, y (3) él para quién lo va a hacer; y es influenciada en momentos concretos por algunos elementos como: la historia de la organización, las preferencias de la gerencia y /o de los propietarios, los factores externos o del entorno, los recursos disponibles, y sus capacidades distintivas.

Para Serna (2008), la misión implica una declaración única de cada organización porque sus principios, sus valores, su visión, la filosofía de sus dueños, los colaboradores y los grupos con los que interactúan en el mercado son para todas las organizaciones diferentes. La misión debe ser claramente formulada, difundida y conocida por todos los colaboradores de la organización para fomentar comportamientos cónsonos con la misma. Por consiguiente, la misión no puede convertirse en formulaciones que aparecen en las oficinas de la compañía. Sino que debe inducir comportamientos y crear compromisos.

De igual manera David (2008, p.10), sostiene que las declaraciones de la misión son:

Expresiones perdurables de los propósitos que distinguen a una empresa de otras empresas similares. Una declaración de la misión identifica el alcance de las operaciones de una empresa en términos del producto y del mercado. Aborda la pregunta básica que enfrentan todos los estrategas: ¿cuál es nuestro negocio? La misión definida como una declaración describe los valores y las prioridades de una empresa.

En consideración a lo expuesto anteriormente, se establece, que debe existir una misión donde se manifiesten las actividades inherentes a las empresas objeto de estudio. Po lo que es importante que los gerentes se encarguen de su divulgación y su internalización por parte del personal.

Objetivos: David (2008), los define como "como resultados específicos que una empresa intenta lograr para cumplir con su misión básica" (p.11). Los objetivos son indispensables para lograr el éxito de una empresa debido a que establecen la dirección a seguir, ayudan en la evaluación, crean sinergia, revelan prioridades, enfocan la coordinación y proporcionan una base para llevar a cabo con eficacia las actividades de planificación, organización, motivación y control.

Agregan Thompson y Strickland (2007), que un objetivo de organización es el blanco hacia el que se orientan los esfuerzos que lleva a cabo una organización. Unos objetivos claros aportan fundamentos sólidos para la formulación de una estrategia, para la ejecución de la misma y para el planteamiento de la acción, por lo cual deben ser especificados y divulgados en las empresas. 
Por su parte Serna (2008), define los objetivos como "resultados a largo plazo que una organización espera lograr para hacer real la misión y la visión de la empresa o área de negocio"(p.73). Estos objetivos empresariales, junto con las estrategias, son los factores integradores de la tarea de la alta gerencia $y$, por tanto, deberán reflejarse en los planes funcionales y operativos de cada unidad estratégica de negocio.

La investigadora considera los objetivos en términos de propósitos a lograr, tales objetivos deben ser revisados para verificar si los mismos se consideran en la actividad de las empresas mixtas petroleras del municipio Maracaibo.

Estrategia: En el criterio de Mintzberg (2008), la estrategia comprende el conjunto de acciones integradas que definen un curso de acción o guía para enfrentar una situación. Por ello, explica que la estrategia debe ser definida a través de la integración y complementariedad de sus distintas acepciones: como plan, pauta, táctica, posición y perspectiva.

En el mismo orden de ideas para David (2008), "son los medios por los cuales se logran los objetivos a largo plazo"(p.12). Las estrategias de negocios incluyen la expansión geográfica, la diversificación, la adquisición, el desarrollo de productos, la penetración en el mercado, la reducción de costos, la enajenación, la liquidación y las empresas conjuntas.

Las estrategias son acciones potenciales que requieren decisiones de parte de la gerencia y de recursos de la empresa. Además, las estrategias afectan las finanzas a largo plazo de una empresa, por lo menos durante cinco años, orientándose así hacia el futuro. Las estrategias producen efectos en las funciones y divisiones de la empresa, y exigen que se tomen en cuenta tanto los factores externos como los factores internos que enfrenta la empresa.

En este sentido Serna (2008, p.73), argumenta que las estrategias son "las acciones que deben realizarse para mantener y soportar el logro de los objetivos de la organización y de cada unidad de trabajo y así hacer realidad los resultados esperados al definir los proyectos estratégicos". Las estrategias son, entonces, las que permiten lograr y hacer realidad cada objetivo y cada proyecto estratégico.

Tomando en cuenta las definiciones anteriores, se sostiene que a pesar de las diversas estrategias de acción existentes, lo más sustancial es que la empresa seleccione aquellas estrategias que más favorezcan el cumplimiento de la visión, misión y objetivos corporativos. Además, se manifiesta que las empresas mixtas petroleras del municipio Maracaibo deben formular y evaluar las estrategias ya que estas representan el medio para superar las debilidades, amenazas, y para aprovechar las oportunidades y fortalezas. 


\section{Ejecución de estrategias}

La ejecución de la estrategia requiere que una empresa establezca planes de acción, diseñe políticas, motive a los empleados y distribuya los recursos de tal manera que se ejecuten las estrategias formuladas; la implementación de la estrategia incluye el desarrollo de una cultura que apoye las estrategias, la creación de una estructura de organización eficaz, la orientación de las actividades, la preparación de presupuestos, la creación y la utilización de sistemas de información y la vinculación de la compensación de los empleados con el rendimiento de la empresa.

Thompson y Strickland (2007) agregan que una vez seleccionada, es necesario iniciar la acción que la ponga en movimiento, para que produzca resultados efectivos y eficientes. Esta actividad se sustenta sobre el trabajo que desarrollan de los clientes internos, la valorización de los recursos; financieros, técnicos, materiales y logísticos necesarios en cada caso, para que la estrategia produzca los resultados esperados de manera oportuna.

Para los autores mencionados, el desarrollo de la actividad comentada, requiere de competencia gerencial para afrontar el cambio e innovar de manera adecuada u oportuna, para poder poner y mantener en marcha la estrategia, ejecutarla en forma eficiente garantizar la obtención de los resultados esperados.

Igualmente, Certo (2008) afirma que la ejecución de estrategias ayuda a la empresa a asignar sus recursos, a sacar provecho de su potencialidad relativa y mitigar sus debilidades, a explotar los cambios que se proyectan en el ambiente y a neutralizar las posibilidades de sus competidores.

La ejecución de las estrategias es la fase de acción propiamente dicha, en la cual entra en funcionamiento las acciones planificadas, lo cual implica la necesidad de considerar diversas acciones en las empresas mixtas, incluyendo los siguientes elementos:

Fijación de metas: De acuerdo con David (2008) comprende el proceso de definir los pasos específicos a realizar para lograr la estrategia. Las metas establecen las acciones en menor escala que deben alcanzarse para llegar a los objetivos macro de la planificación estratégica. Las empresas deben fijar las metas administrativas y operativas en la ejecución de las estrategias e informarlas al personal para lograr en la ejecución de la estrategia.

Al respecto, Thompson y Strickland (2007) agregan que en la planificación estratégica de las organizaciones, es esencial que los expertos o analistas de las mismas elaboren un conjunto de metas específicas, las cuales identifican el orden de prioridad de las acciones que deben emprenderse. Comprende el proceso de definir los pasos específicos a realizar para lograr la estrategia. Las metas establecen las acciones en menor escala que deben alcanzarse para llegar a los objetivos macro de la planificación estratégica.

Serna (2008) afirma que las metas son puntos de referencia o aspiraciones que las organizaciones deben lograr, con el objeto de alcanzar en el futuro objetivos a un plazo más largo. Su importancia radica en que permite sistematizar las acciones a seguir e ir evaluando los logros parciales de la estrategia global. 
Así las cosas, la investigadora considera las metas como pasos de menor dimensión, los cuales se estructuran con el propósito de lograr los objetivos planteados en las empresas mixtas.

Fijación de políticas: Para David (2008) comprende el proceso de definir los lineamientos generales que apoyan la estrategia. Las políticas deben ser difundidas entre el personal y la gerencia debe velar por el cumplimiento de las políticas en la ejecución de las estrategias que permiten lograr el crecimiento sostenido.

Thompson y Strickland (2007) agregan que son las acciones de los trabajadores en función de lograr la estrategia planteada en las organizaciones. Estos lineamientos empresariales deben ser emanados de la actividad de los agentes organizacionales y deben ser manejados con amplio conocimiento por el personal que configura la actividad estratégica de las empresas.

Del mismo modo, Serna (2008) expresa que las políticas incluyen la forma por medio de la cual las metas fijadas van a lograrse o las pautas establecidas para respaldar esfuerzos con el objeto de lograr las metas ya definidas. Estas políticas deben ser difundidas para ser utilizadas como referencia por el personal.

Desde estas perspectivas, las políticas son lineamientos generales que conducen o guían al trabajador, por lo cual deben ser conocidas por éstos; en este caso aplicado a las empresas mixtas objeto de estudio.

Asignación de recursos: David (2008) indica que consiste en la definición de los recursos económicos y humanos requeridos para ejecutar la estrategia. Explica que en la ejecución de las estrategias se debe incluye el análisis de los recursos humanos requeridos, los recursos económicos requeridos y se debe velar por el uso racional de los recursos asignados para la ejecución de la estrategia.

Asimismo, Thompson y Strickland (2007) agregan que los recursos de las organizaciones para apoyar la estrategia son de diversa índole, siendo lo pertinente que las empresas en particular se orienten a disponer de forma oportuna del personal o recurso humano necesario, así como de los bienes económicos o fuentes de financiamiento para apoyar la ejecución de estos proyectos.

Por su parte, Serna (2008) expresa que los recursos humanos y materiales son esenciales para darle la viabilidad necesaria a la estrategia por cuanto ellos son la inversión en capital humano para llevar hacia adelante el emprendimiento de los objetivos trazados, así como recursos operativos y financieros.

Los recursos son considerados en este estudio, desde la perspectiva de David (2008) a los fines de valorar si las empresas mixtas cuentan con este soporte esencial en la planificación estratégica.

\section{Evaluación de la estrategia}

Para David (2008) es la tercera fase de la planificación estratégica dirigida a realizar una valoración del desempeño de la estrategia, a fin de asumir las medidas correctivas. Es necesario analizar los factores internos y externos que representa las bases de sus estrategias actuales, hacer 
una evaluación de estrategia empresarial, en tanto una firma debe medir el desempeño de la organización y se debe comparar el proceso real con el progreso previamente planificado de la empresa, con respecto al logro de las metas y objetivos previamente establecidos, a los fines de determinar los logros alcanzados.

Strickland y Thompson (2007) opinan que esta actividad implica el direccionamiento estratégico y el desempeño de la estrategia considerando los resultados obtenidos, la influencia de las condiciones cambiantes del entorno, los procesos de innovación y las oportunidades que se ofrezca el mercado. La evaluación proactiva permanente, es necesaria; por que la visión, la misión (razón de ser del negocio), los objetivos, la estrategia y el enfoque de la empresa a la implantación nunca terminan de evolucionar.

Por su parte, Certo (2008), expresan que la evaluación abarca el control estratégico, que es un tipo especial de control organizativo que consiste en el seguimiento y evaluación del proceso de administración estratégica con el fin de mejorarlo para asegurar su funcionamiento. Para llevar a cabo esta tarea de control de manera satisfactoria los administradores han de entender la importancia de las auditorías estratégicas. Por otra parte, los directores de las empresas e instituciones deben intuir la complejidad de su sistema de información de administración, así como de gestión aplicado, y la manera en que este sistema puede servir de complemento al proceso de control estratégico.

A partir de los enfoques descritos, se coincide al referir la importancia de realizar una valoración de la estrategia como una medida de control del rendimiento obtenido por las empresas en la actividad de planificación ejecutada. Para este estudio, se consideran los planteamientos de David (2008) quien destaca la importancia de la evaluación de estrategias e incluye los siguientes aspectos:

Medición de resultados: De acuerdo con David (2008), son las acciones enfocadas en comparar el proceso real con el progreso previamente planificado de la empresa, con respecto al logro de las metas y objetivos previamente establecidos. Para ello, la empresa debe medir los resultados de la estrategia en función de las metas trazadas, evaluar el nivel de logro de los objetivos e informar al personal los resultados de la evaluación de la estrategia.

Thompson y Strickland (2007), señalan que la evaluación estratégica amerita un proceso de monitoreo, porque produce decisiones que pueden tener consecuencias significativas a largo plazo. Una decisión estratégica equivocada puede provocar graves daños y puede ser muy difícil de revertir, por no decir que imposible.

Del mismo modo, Serna (2008) expresa que la efectividad del monitoreo de la gestión estratégica representa la vía para competir activamente dentro de un entorno de turbulencia. Agrega que solo a través del diagnóstico de la gestión estratégica es posible concretar las estrategias más idóneas, formulándolas y ejecutándolas para lograr el éxito de las organizaciones. 
Considerando lo antes mencionado, es importante valorar la ejecución de las acciones estratégicas emprendidas por las empresas, porque sirve de base para tomar decisiones acertadas sobre el rumbo de las empresas; destacándose el empleo de los planteamientos de David (2008) a los efectos de medirlo en el entorno de las empresas mixtas.

Medidas correctivas: David (2008) indica que son acciones para corregir las variables que pueden afectar el desempeño de la estrategia. Se deben tomar medidas correctivas, requeridas para mejorar la posición estratégica de la firma tanto externa como internamente. Las empresas exitosas se anticipan y se adaptan a través de medidas correctivas a los cambios en forma rápida y efectiva.

En este proceso, el autor afirma que se deben tomar medidas correctivas, requeridas para mejorar la posición estratégica de la firma tanto externa como internamente. La evaluación de estrategia es una etapa crítica en el proceso de gestión estratégica debido a que los factores internos y externos sufren cambios. Las empresas exitosas se anticipan y se adaptan a los cambios en forma rápida y efectiva.

Thompson y Strickland (2007), agregan que es necesario considerar la valoración de las estrategias para definir los nuevos cursos de acción, así como acometer las medidas correctivas. Esta valoración puede realizarse considerando los efectos de la estrategia sobre los factores internos y externos.

También Serna (2008) resalta la necesidad de evaluar las estrategias de las empresas, indicando que las mismas se ajustan en forma cambian de forma significativa. La evaluación de la estrategia es necesaria debido a que el éxito presente no es garantía para el éxito futuro.

Es el caso de las empresas mixtas, estas necesitan mejorar la comprensión de su estrategia, comunicarla mejor y hacer un adecuado seguimiento. Para ello, se requieren mejores sistemas de dirección que proporcionen la información y los incentivos adecuados, permitan controlar el cumplimiento, aseguren la comunicación, la coordinación y el control de las personas que actúan en la organización.

Por ello, se consideran básicos los planteamientos de David (2008) en torno a la formulación, ejecución y evaluación de las estrategias, tomando como referencia los aspectos integrantes de cada una de estas etapas para medirlas en las organizaciones del sector textil, a los fines de verificar la forma en la cual vienen ejecutando este proceso estratégico.

\section{MÉTODO}

$\mathrm{M}$ etodológicamente fue tipificada como una investigación descriptiva, con un diseño no experimental, transeccional y de campo. La población del estudio estuvo conformada por seis empresas mixtas del municipio Maracaibo del estado Zulia, Venezuela, activas al momento de la recolección de la información. Cuyos criterios de inclusión para los sujetos 
informantes son: (a) Trabajar en una empresa mixta; (b) Ocupar una posición gerencial en el área de planificación; (c) Antigüedad en el cargo mayor o igual a 5 años; d) Experiencia acumulada mayor o igual a 3 años.

La técnica de recolección de datos utilizada fue la encuesta y el instrumento utilizado un cuestionario con cinco alternativas de respuesta, estructurado por 31 ítems para las etapas de la planificación estratégica, se utilizó el juicio de expertos para la validez del mismo y el coeficiente Alfa Cronbach para la confiabilidad, cuyo resultado fue 0,96.

La información fue procesada a través de la estadística descriptiva específicamente mediante la media aritmética. Para ello, se realizó un baremo con la finalidad de analizar e interpretar los datos obtenidos de la aplicación del cuestionario. El mismo se diseñó considerando los puntajes máximos asignados a cada reactivo, además de los atributos establecidos, tal como se muestra en el cuadro 1.

Cuadro 1. Categoría de análisis para la interpretación del promedio

\begin{tabular}{|c|c|c|c|}
\hline DIMENSIÓN & SUB - DIMENSIÓN & RANGO & NIVEL DE RESPUESTA \\
\hline & Formulación de & $4.20 \leq 5.00$ & Muy alta presencia \\
\hline & estrategias & $3.40<4.20$ & Alta presencia \\
\hline & & $2.60<3.40$ & Moderada presencia \\
\hline & & $1.80<2.60$ & Baja presencia \\
\hline & & $1.00<1.80$ & No hay presencia \\
\hline & Ejecución de & $4.20 \leq 5.00$ & Muy alta presencia \\
\hline & estrategias & $3.40<4.20$ & Alta presencia \\
\hline \multirow[t]{8}{*}{ Etapas } & & $2.60<3.40$ & Moderada presencia \\
\hline & & $1.80<2.60$ & Baja presencia \\
\hline & & $1.00<1.80$ & No hay presencia \\
\hline & Evaluación de & $4.20 \leq 5.00$ & Muy alta presencia \\
\hline & estrategias. & $3.40<4.20$ & Alta presencia \\
\hline & & $2.60<3.40$ & Moderada presencia \\
\hline & & $1.80<2.60$ & Baja presencia \\
\hline & & $1.00<1.80$ & No hay presencia \\
\hline
\end{tabular}

\section{RESULTADOS}

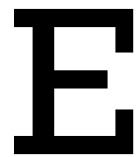
n la tabla 1 se resumen los resultados relacionados con la sub dimensión formulación de estrategias, con un promedio de 3,35 lo que la ubicó en una categoría moderada presencia indicando que moderadamente las empresas mixtas en estudio incluyó en su proceso de planificación estratégica la determinación de las fortalezas y debilidades internas, la identificación de las oportunidades y amenazas externas de una organización, la creación de una visión y misión, 
el establecimiento de objetivos así como la generación de estrategias. Así, se representa una oportunidad de mejora por cuento que para bien o para mal, esta etapa del proceso puede dar lugar a una ventaja competitiva sostenible.

Tabla 1. Indicadores de la sub-dimensión formulación de estrategias

\begin{tabular}{|c|c|c|c|c|}
\hline INDICADOR & $\mathbf{N}^{\circ}$ & ITEMS & $\overline{\boldsymbol{X}}$ & CATEGORIA \\
\hline \multirow{5}{*}{ Análisis interno } & 1 & $\begin{array}{l}\text { Evalúa los atributos de la empresa que favorecen el } \\
\text { logro de los objetivos. }\end{array}$ & 3,67 & Alta presencia \\
\hline & 2 & $\begin{array}{l}\text { Examina las condiciones que dificultan el éxito de la } \\
\text { empresa }\end{array}$ & 4,00 & Alta presencia \\
\hline & 3 & $\begin{array}{l}\text { Analiza la factibilidad económica para la inversión de } \\
\text { capital. }\end{array}$ & 3,83 & Alta presencia \\
\hline & 4 & $\begin{array}{l}\text { Conoce la capacidad tecnológica con la que dispone } \\
\text { para afrontar las exigencias de producción. }\end{array}$ & 4,08 & Alta presencia \\
\hline & & INDICADOR & 3,90 & Alta presencia \\
\hline \multirow{4}{*}{ Análisis externo } & 5 & $\begin{array}{l}\text { Consideran las nuevas tecnologías del mercado que } \\
\text { brindan mejoras al proceso productivo de la empresa }\end{array}$ & 3,08 & Moderada presencia \\
\hline & 6 & $\begin{array}{l}\text { Discuten los factores políticos del entorno que afectan } \\
\text { el rendimiento de las operaciones }\end{array}$ & 3,33 & Moderada presencia \\
\hline & 7 & $\begin{array}{l}\text { Estiman los cambios en la economía global que } \\
\text { afectan a la empresa. }\end{array}$ & 3,25 & Moderada presencia \\
\hline & & INDICADOR & 3,22 & $\begin{array}{l}\text { Moderada } \\
\text { presencia }\end{array}$ \\
\hline \multirow{4}{*}{ Visión } & 8 & Se identifica con la visión de la empresa & 3,50 & Alta presencia \\
\hline & 9 & Divulga al personal la visión de la empresa & 2,50 & Moderada presencia \\
\hline & 10 & Busca el cumplimiento de lo establecido en la visión & 3,08 & Moderada presencia \\
\hline & & INDICADOR & 3,03 & $\begin{array}{l}\text { Moderada } \\
\text { presencia }\end{array}$ \\
\hline \multirow{4}{*}{ Misión } & 11 & Se identifica con la misión de la empresa & 3,33 & Moderada presencia \\
\hline & 12 & Divulga al personal la misión de la empresa & 2,75 & Moderada presencia \\
\hline & 13 & Busca el cumplimiento de lo establecido en la misión & 3,00 & Moderada presencia \\
\hline & & INDICADOR & 3,03 & $\begin{array}{l}\text { Moderada } \\
\text { presencia }\end{array}$ \\
\hline \multirow{4}{*}{ Objetivos } & 14 & Establece objetivos medibles & 3,67 & Alta presencia \\
\hline & 15 & $\begin{array}{l}\text { Traza objetivos que reflejan las prioridades de la } \\
\text { empresa }\end{array}$ & 3,50 & Alta presencia \\
\hline & 16 & Busca el logro de los objetivos & 3,67 & Alta presencia \\
\hline & & INDICADOR & 3,61 & Alta presencia \\
\hline
\end{tabular}




\begin{tabular}{|c|c|c|c|c|}
\hline INDICADOR & $\mathbf{N}^{\circ}$ & ITEMS & $\overline{\bar{X}}$ & CATEGORIA \\
\hline \multirow{4}{*}{ Objetivos } & & Establece objetivos medibles & 3,67 & Alta presencia \\
\hline & 15 & $\begin{array}{l}\text { Traza objetivos que reflejan las prioridades de la } \\
\text { empresa }\end{array}$ & 3,50 & Alta presencia \\
\hline & 16 & Busca el logro de los objetivos & 3,67 & Alta presencia \\
\hline & & INDICADOR & 3,61 & Alta presencia \\
\hline \multirow{5}{*}{ Estrategias } & 17 & $\begin{array}{l}\text { Utiliza la información de los análisis del entorno para } \\
\text { formular líneas de acción }\end{array}$ & 3,17 & Moderada presencia \\
\hline & 18 & $\begin{array}{l}\text { Establece líneas de acción según las prioridades de la } \\
\text { empresa }\end{array}$ & 3,25 & Moderada presencia \\
\hline & 19 & $\begin{array}{l}\text { Estables combinaciones de acciones en forma } \\
\text { simultánea para el logro de los objetivos planteados }\end{array}$ & 3,42 & Alta presencia \\
\hline & & INDICADOR & 3,28 & $\begin{array}{l}\text { Moderada } \\
\text { presencia }\end{array}$ \\
\hline & & SUB.DIMENSION & 3,35 & $\begin{array}{l}\text { Moderada } \\
\text { presencia }\end{array}$ \\
\hline
\end{tabular}

Al detalle, se aprecia una media de 3,90 indicando una alta presencia del análisis interno, de manera que, los resultados demostraron que en el departamento de perforación de las empresas mixtas petroleras del municipio Maracaibo, Venezuela, se evaluaron todas las capacidades internas de la empresa, con la finalidad de conocer la situación de la organización, admitiendo que se cumple la teoría expuesta por David (2008).

Con relación al indicador análisis externo, se evidenció un promedio de 3,22 ubicándolo en la categoría de moderada presencia. En atención a esto, se aprecia que las empresas objeto de estudio, moderadamente consideró que el éxito de la planificación depende en parte de la organización y el entorno en el cual esta compite, y es determinado por medio del análisis externo, validando parcialmente lo planteado por Serna (2008).

En cuanto al indicador visión, los resultados revelaron un promedio de 3,03 ubicándola en la categoría de moderada presencia. En este sentido, los resultados si bien revelan que en las empresas mixtas petroleras del municipio Maracaibo en Venezuela, identificaron que en la visión es importante la necesidad de que sus fundamentos sean difundida en la organización y reconocida, por ello requiere un sistema de difusión que la haga conocer y comprender por todos los miembros del departamento de perforación, otorgando parcial congruencia con lo expuesto por Serna (2008).

Respecto al indicador misión, los resultados lo situaron en una media de 3,03, ubicándola en una categoría de moderada presencia. Esto demostraron que en las empresas bajo estudio, moderadamente se consideraron, el diseño y divulgación de la razón de ser de la organización. 
Estos bajos puntajes difieren con David (2008), quien establece que la misión es el fundamento para la planificación de la gestión.

Siguiendo con los indicadores de las etapas del proceso de la planificación estratégica, se tuvo los objetivos, cuya media de acuerdo a los sujetos encuestados se ubicaron en 3,61 lo cual corresponde a una categoría de alta presencia, así las cosas, en las empresas bajo estudio crearon objetivos medibles y realistas con la finalidad de consolidar la planificación estratégica, así como el interés en su divulgación al personal para que se tengan presente las metas a cumplir, dándose validez así, a lo expuesto por David (2008).

En cuanto al indicador estrategias, los resultados revelaron un promedio de 3,28, ubicándolo en la categoría de moderada presencia, de manera que en las empresas analizadas las estrategias formuladas permiten el cumplimiento de la visión, misión y objetivos corporativos, coincidiendo en parte con la teoría de Serna (2008).

Seguidamente, en la tabla 2, se resumen los resultados relacionados con la sub dimensión ejecución de estrategias, con un promedio de 3,12 lo que la ubicaron en una categoría moderada presencia indicando que moderadamente las empresas mixtas en estudio incluyen en su proceso de planificación estratégica fijación de metas, políticas y asignación de recursos.

Esta fase de la ejecución de estrategias se enfocó en considerar la acción propiamente dicha, en la cual entra en funcionamiento las acciones planificadas, lo cual implica la necesidad de establecer diversas acciones en las empresas mixtas. Al respecto, David (2008), afirma que la ejecución de la estrategia requiere que una empresa establezca planes de acción, diseñe políticas, motive a los empleados y distribuya los recursos de tal manera que se ejecuten las estrategias formuladas.

Tabla 2. Indicadores de la sub-dimensión ejecución de estrategias

\begin{tabular}{|c|c|c|c|c|}
\hline INDICADOR & $\mathbf{N}^{\circ}$ & ITEMS & $\bar{X}$ & CATEGORIA \\
\hline \multirow{4}{*}{$\begin{array}{l}\text { Fijación de } \\
\text { metas }\end{array}$} & 20 & $\begin{array}{l}\text { Define los pasos específicos a realizar para lograr la } \\
\text { estrategia }\end{array}$ & 3,00 & Moderada presencia \\
\hline & 21 & $\begin{array}{l}\text { Identifica el orden de prioridad de las acciones que } \\
\text { deben emprenderse }\end{array}$ & 3,25 & Moderada presencia \\
\hline & 22 & $\begin{array}{l}\text { Sistematiza las acciones a seguir para ir evaluando los } \\
\text { logros parciales de la empresa }\end{array}$ & 3,17 & Moderada presencia \\
\hline & & INDICADOR & 3,14 & $\begin{array}{l}\text { Moderada } \\
\text { presencia }\end{array}$ \\
\hline
\end{tabular}




\begin{tabular}{|c|c|c|c|c|}
\hline INDICADOR & $\mathbf{N}^{\circ}$ & ITEMS & $\overline{\bar{X}}$ & CATEGORIA \\
\hline & 23 & $\begin{array}{l}\text { Define los lineamientos generales que apoyan la } \\
\text { estrategia }\end{array}$ & 3,17 & Moderada presencia \\
\hline \multirow[t]{3}{*}{$\begin{array}{l}\text { Fijación de } \\
\text { políticas }\end{array}$} & 24 & $\begin{array}{l}\text { Establece las pautas necesarias para lograr las metas } \\
\text { ya definidas }\end{array}$ & 2,75 & Moderada presencia \\
\hline & & INDICADOR & 2,96 & $\begin{array}{l}\text { Moderada } \\
\text { presencia }\end{array}$ \\
\hline & 25 & $\begin{array}{l}\text { Establece los recursos económicos requeridos para } \\
\text { ejecutar la estrategia }\end{array}$ & 3,17 & Moderada presencia \\
\hline \multirow[t]{3}{*}{$\begin{array}{l}\text { Asignación de } \\
\text { recursos }\end{array}$} & 26 & $\begin{array}{l}\text { Establece los recursos humanos requeridos para } \\
\text { ejecutar la estrategia }\end{array}$ & 3,33 & Moderada presencia \\
\hline & & INDICADOR & 3,25 & $\begin{array}{l}\text { Moderada } \\
\text { presencia }\end{array}$ \\
\hline & & SUB.DIMENSION & 3,12 & $\begin{array}{l}\text { Moderada } \\
\text { presencia }\end{array}$ \\
\hline
\end{tabular}

En específico, se observó para el indicador fijación de metas, un promedio de 3,14, ubicándola en la categoría de moderada presencia. Esta posición de moderada representa para las empresas mixtas objeto de estudio oportunidades de mejora al trazar metas con el objeto de alcanzar en el futuro objetivos a un plazo más largo, de manera que existe parcial congruencia con lo expuesto por Thompson y Strickland (2007), para quienes es esencial que los expertos o analistas de las mismas elaboren un conjunto de metas específicas, las cuales identifican el orden de prioridad de las acciones que deben emprenderse.

Para el indicador fijación de políticas, se evidenció un promedio de 2,96 ubicándolo en la categoría de moderada presencia, de esta manera, la fijación de políticas debe ser enmarcada en la actividad de los agentes organizacionales y deben ser manejados con amplio conocimiento por el personal que configura la actividad estratégica de las empresas mixtas petroleras del municipio Maracaibo, Venezuela. Tal como lo expresa David (2008), al considerar que la fijación de políticas comprende el proceso de definir los lineamientos generales que apoyan la estrategia.

En lo concerniente al indicador asignación de recursos, se aprecia una media de 3,25 ubicándola en una categoría de moderada presencia. Los resultados obtenidos demuestran que en el departamento de perforación de las empresas mixtas petroleras del municipio Maracaibo, Venezuela, no siempre se asignan los esenciales para darle la viabilidad necesaria a la estrategia. De manera que, estos resultados, parcialmente coinciden con lo expuesto por Thompson y Strickland (2007), para quienes los recursos de las organizaciones deben apoyar la estrategia que permitan la ejecución de proyectos.

Continuando con el análisis de los resultados, se puede apreciar en la tabla 3, el resumen para la sub - dimensión evaluación de estrategias, perteneciente a las etapas del proceso de planificación estratégica presentes en el departamento de perforación de las empresas mixtas 
petroleras del municipio Maracaibo, Venezuela, el cual muestra un valor de 3,14, reflejando de acuerdo a la opinión de los encuestados que esta etapa se cumple moderadamente, por lo tanto representa una oportunidad de mejora que le permita a las empresas objeto de estudio valoración el desempeño de la estrategia, a fin de asumir las medidas correctivas.

Según los hallazgos obtenidos, se observó que en las empresas analizadas es necesario hacer seguimiento y evaluación del proceso de administración estratégica con el fin de mejorarlo para asegurar su funcionamiento. Al respecto David (2008) opina que esta etapa es la tercera fase de la planificación estratégica dirigida a realizar una valoración del desempeño de la estrategia, a fin de asumir las medidas correctivas.

Tabla 3. Indicadores de la sub-dimensión evolución de estrategias

\begin{tabular}{|c|c|c|c|c|}
\hline INDICADOR & $\mathbf{N}^{\circ}$ & ITEMS & $\overline{\boldsymbol{X}}$ & CATEGORIA \\
\hline \multirow{4}{*}{$\begin{array}{l}\text { Medición de los } \\
\text { resultados }\end{array}$} & 27 & $\begin{array}{l}\text { Realiza las acciones necesarias para comparar el } \\
\text { proceso real con el progreso previamente } \\
\text { planificado de la empresa }\end{array}$ & 3,50 & Alta presencia \\
\hline & 28 & $\begin{array}{l}\text { Mide los resultados de la estrategia en función de } \\
\text { las metas trazadas }\end{array}$ & 3,33 & $\begin{array}{l}\text { Moderada } \\
\text { presencia }\end{array}$ \\
\hline & 29 & $\begin{array}{l}\text { Verifica que estrategias implementadas son las } \\
\text { más idóneas }\end{array}$ & 2,75 & $\begin{array}{l}\text { Moderada } \\
\text { presencia }\end{array}$ \\
\hline & & & 3,19 & $\begin{array}{l}\text { Moderada } \\
\text { presencia }\end{array}$ \\
\hline \multirow{4}{*}{$\begin{array}{l}\text { Medidas } \\
\text { correctivas }\end{array}$} & 30 & $\begin{array}{l}\text { Realiza acciones para corregir las variables que } \\
\text { pueden afectar el desempeño de la estrategia }\end{array}$ & 3,08 & $\begin{array}{l}\text { Moderada } \\
\text { presencia }\end{array}$ \\
\hline & 31 & $\begin{array}{l}\text { Define continuamente los nuevos cursos de } \\
\text { acción }\end{array}$ & 3,08 & $\begin{array}{l}\text { Moderada } \\
\text { presencia }\end{array}$ \\
\hline & & INDICADOR & 3,08 & $\begin{array}{l}\text { Moderada } \\
\text { presencia }\end{array}$ \\
\hline & & SUB.DIMENSION & 3,14 & $\begin{array}{l}\text { Moderada } \\
\text { presencia }\end{array}$ \\
\hline
\end{tabular}

Con relación al indicador medición de resultados, se evidenció un promedio de 3,19, ubicándola en la categoría de moderada presencia. En atención a esto, se aprecia por medio de los resultados que moderadamente las empresas mixtas petroleras del municipio Maracaibo miden los resultados de la estrategia en función de las metas trazadas, evalúan el nivel de logro de los objetivos e informan al personal los resultados de la evaluación de la estrategia. Lo que, moderadamente coincide con lo expuesto por Thompson y Strickland (2007), quienes señalan que la evaluación estratégica amerita un proceso de monitoreo. 
En cuanto al indicador medidas correctivas, los resultados revelan un promedio de 3,08 ubicándola en la categoría de moderada presencia. Esta situación de moderada presencia, refuerza la necesidad de valorar las estrategias para definir los nuevos cursos de acción, así como acometer las medidas correctivas. Esta valoración puede realizarse considerando los efectos de la estrategia sobre los factores internos y externos, tal como lo plantean Thompson y Strickland (2007).

Finalmente, en la tabla 4, se resumen los resultados relacionados con la dimensión etapas del proceso, que permitió medir la variable planificación estratégica, con un promedio de 3,20 que la ubicó en una categoría de moderada presencia, indicando que moderadamente las empresas mixtas analizadas conocen e implementan las etapas del proceso de la planificación estratégica, en tal sentido, las estrategias inicialmente se formulan sobre las bases de su misión y visión, una vez formuladas se pasa a la fase de acción, asignando los recursos necesarios por medio de políticas bien definidas que buscan integrar a todos los departamentos hacia el logro de los objetivos, para finalmente proceder a evaluar los resultados obtenidos, asimismo se toman las acciones correctivas pertinentes.

Tabla 4: Etapas del proceso de planificación estratégica

\begin{tabular}{lcc}
\hline \multicolumn{1}{c}{ INDICADOR } & PROMEDIO & CATEGORÍA \\
\hline Formulación de estrategias & 3,35 & Moderada presencia \\
Ejecución de estrategias & 3,12 & Moderada presencia \\
Evaluación de estrategias & 3,14 & Moderada presencia \\
\multicolumn{1}{c}{ PROMEDIO DIMENSIÓN } & $\mathbf{3 , 2 0}$ & MODERADA PRESENCIA \\
\hline
\end{tabular}

Este conjunto de acciones se ajusta con lo planteado por David (2008), quien indica que la planificación estratégica es el arte y la ciencia de formular, implementar así como evaluar decisiones multifuncionales que le permitan a una organización lograr sus objetivos, para tal propósito se deben integrar de manera armónica la administración, el marketing, las finanzas y la contabilidad, siendo su finalidad poder aprovechar las oportunidades existentes y crear nuevas oportunidades con miras hacia el futuro.

\section{CONCLUSIONES}

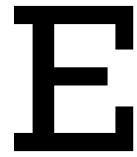
n relación al objetivo, dirigido a identificar las etapas del proceso de planificación estratégica en las empresas mixtas petroleras del municipio Maracaibo, los resultados alcanzados permiten concluir que moderadamente las empresas mixtas analizadas conjugan estas etapas, a pesar de la importancia que tiene la planificación estratégica para ellas. 
Según los resultados descritos, se evidenció que se presentan dificultades a la hora de aplicarla, debido fundamentalmente a que no siempre se conocen los factores externos, su visión y misión, ni están claramente definidos sus estrategias, lo cual da lugar a ambigüedades e inseguridad con respecto al seguimiento y evaluación de los planes.

En este sentido, es importante que las empresas mixtas analizadas cumplan con una triple función: de diagnóstico, para detectar los aciertos y desaciertos de su praxis, con el propósito de diseñar la capacitación o los correctivos necesarios para coadyuvar en la erradicación de sus deficiencias; de reflexión, a fin de que se internalice qué es lo que está realizando y cómo se está haciendo, todo ello en función de las percepciones del entorno y de la comunidad en general; y por último, se podría decir que tiene una función de crecimiento o maduración, ya que como resultado del proceso de planificación, se torna capaz de autoevaluar permanentemente su desempeño y llega a una meta-comprensión de lo que no se sabe y lo que se necesita comprender.

No obstante, hacer el plan no es suficiente para garantizar el éxito a largo plazo de las empresas mixtas petroleras del municipio Maracaibo, este debe ir acompañado de un instrumento que permita sistematizar desde los niveles operativos, los objetivos y estrategias definidos desde la planificación estratégica.

\section{REFERENCIAS}

Borrell, F. (2003). Cómo Trabajar en Equipo. Ediciones Gestión Barcelona

Certo, S. (2008). Dirección Estratégica. Editorial Trillas. México

Chiavenato, I.(2005). Gestión del talento humano. Editorial McGraw-Hill .México

David, F. (2008). Conceptos de administración estratégica. 11a ed., Ed. Pearson Prentice Hall. México DF, México

Goodstein, L.; Nolan, T., y Pfeiffer, J. (2006). Planeación estratégica aplicada. Primera edición. McGraw Hill. Santa Fe Bogotá, Colombia
Hax, A., y Majluf, N. (2004). Estrategia para el liderazgo competitivo. Ediciones Granica S. A. Argentina

Mintzberg, H.(2008).El Proceso Estratégico. Editorial Pearson Educación. México

Münch, L. y García, J. (2008). Fundamentos de Administración. Trillas. México

Serna, H. (2008). Gerencia Estratégica. 10a Edición. Editorial Panamericana. Colombia

Thompson, A., y Strickland, A. (2007). Administración Estratégica. Textos y Casos. $13^{\circ}$ Edición. M McGraw Hill. Colombia 